\title{
Autofluorescence in Parathyroidectomy: Signal Intensity Correlates with Serum Calcium and Parathyroid Hormone but Routine Clinical Use is Not Justified
}

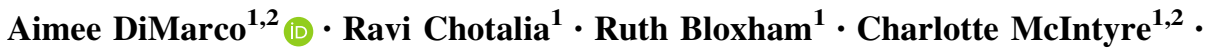 \\ Neil Tolley ${ }^{1,2} \cdot$ F. Fausto Palazzo ${ }^{1,2}$
}

Published online: 8 February 2019

(C) The Author(s) 2019

\begin{abstract}
Background The inability to identify the pathological gland at surgery results in failure to cure hyperparathyroidism in $2-5 \%$. The poorly understood characteristic of parathyroid tissue to manifest autofluorescence (AF) under nearinfrared (NIR) light has been promoted as an intraoperative adjunct in parathyroid surgery. This study sought to explore potential clinical correlates for AF and assess the clinical utility of AF in parathyroid surgery.

Methods Consecutive patients undergoing parathyroid surgery for primary and renal disease were included. NIR imaging was used intraoperatively and the degree of AF of parathyroid glands graded by the operating surgeon. Variables assessed for correlation with AF were: pre-operative serum calcium and PTH, SestaMIBI positivity, gland weight and histological composition.

Results Ninety-six patients underwent parathyroidectomy over an 8-month period: 49 bilateral explorations, 41 unilateral and 6 focussed lateral approaches: 284 potentially 'visualisable' glands in total. Two hundred and fiftyseven glands $(90.5 \%)$ were visualised with NIR. Correlation was found between the degree of fluorescence and preoperative serum calcium and PTH, but not between gland weight and SestaMIBI positivity. In those with renal hyperparathyroidism, a predominance of oxyphil cells correlated with increased AF.

Conclusion Autofluorescence intensity correlates with serum calcium, PTH and gland composition. Further refinements would be required for this information to be of value in a clinical setting. Improvements allowing NIR to visualise the additional $9.5 \%$ of parathyroids and overcome the variation in signal intensity due to depth of access are required for the routine adoption of this technology. At present, its routine use in a clinical setting cannot be justified.
\end{abstract}

\section{Introduction}

'Autofluorescence' (AF) was first described in 1838 and represents the emission of light in the near-infrared (NIR) spectrum by a biological substrate, following excitation by light of a certain wavelength [1]. This property has been

F. Fausto Palazzo

f.palazzo@imperial.ac.uk

1 Department of Endocrine Surgery, Hammersmith Hospital, Du Cane Road, London W12 0HS, UK

2 Department of Surgery and Cancer, Imperial College London, London, UK exploited in several clinical specialties including dermatology, ophthalmology and oncological surgery [2-8]. AF in parathyroid tissue was first demonstrated in vivo in humans in 2011 [9] and has been shown to be distinct from thyroid autofluorescence (around 3 times brighter), typically occurring in the $800-950 \mathrm{~nm}$ wavelength (after excitation at around $785 \mathrm{~nm}$ ). It has therefore been considered as a possible intraoperative adjunct in localisation of the parathyroid glands during neck endocrine surgery [9-11]. This generated considerable interest as the detection of parathyroid tissue intraoperatively is dependent upon visual inspection by the operating surgeon with outcomes being commensurate with experience $[12,13]$. 
Several studies have also sought to use AF with an intravenous fluorophore, such as indocyanine green (ICG), to demonstrate the vascularity of the glands as a surrogate marker of parathyroid function following thyroidectomy, with the hypothesis that its use will prevent post-thyroidectomy hypoparathyroidism [14, 15]. In parathyroidectomy (PTx) for hyperparathyroidism, the challenge is to ensure identification of all parathyroid tissues, pathological and normal, as failure to do so results in persistent hyperparathyroidism. Given that the rate of failure to cure in the first time parathyroid surgery can be as low as $2 \%$, achieving a further reduction would require a significant improvement in either pre- or intraoperative localisation adjuncts. An understanding of the characteristics of AF in physiological and pathological parathyroid tissues is required if it is to be used with benefit. However, although multiple candidates have been proposed, the endogenous fluorophore/s responsible for parathyroid autofluorescence is/are not yet known [1,9]. The purpose of this study was to explore the potential clinical correlates for parathyroid AF and assess the clinical utility of $\mathrm{AF}$ in parathyroid surgery.

\section{Methods}

The aims of this study were twofold: (1) to assess the reliability of parathyroid AF in surgery for hyperparathyroidism (HPT) and (2) to establish whether a correlation exists between the intensity of AF and any of the following variables-pre-operative serum calcium or parathyroid hormone (PTH), SestaMIBI positivity, gland weight and histological composition of the gland.

Following the approval by the ethical review board, a prospective study was conducted of consecutive patients undergoing parathyroid surgery in a single tertiary referral teaching hospital, performing over 650 endocrine surgical procedures (thyroid, parathyroid and adrenal) per annum. Inclusion criteria were: all patients undergoing parathyroid surgery (bilateral exploration, unilateral exploration and focussed lateral approach) for primary or secondary disease. Primary hyperparathyroidism (pHPT) was diagnosed on the basis of an elevated serum calcium in the presence of an inappropriately unsuppressed PTH or normocalcaemic hyperparathyroidism, a normal calcium and inappropriately high PTH. Urinary calcium/creatinine clearance ratio was calculated from a $24-\mathrm{h}$ urine collection in all patients to exclude benign familial hypocalciuric hypercalcaemia [16]. The indications for intervention in pHPT were clinical symptoms or the loose application of the NIH criteria in asymptomatic disease [17]. Secondary hyperparathyroidism was due to chronic renal failure requiring dialysis in all cases, and the KDIGO guidelines were used to determine the timing of intervention, based on the principle that the severity of disease predicted irreversibility post-transplant [18]. Patients with pHPT underwent dual localisation studies pre-operatively with ultrasound and SestaMIBI. Additional imaging with 4DCT and/or venous sampling/parathyroid angiography was performed in patients with recurrent/persistent pHPT. Those with concordant imaging went on to focussed lateral approach PTx and non-concordant/single modality positive imaging to unilateral exploration with intraoperative PTH estimation (StatIOPTH, Future Diagnostics) guided by the Miami criteria [19]. Unlocalised primary disease and renal HPT were managed with bilateral exploration. There were no exclusion criteria.

The AF device used was the 'Fluobeam ${ }^{\circledR} 800$ ' (Fluoptics, Grenoble, France), because it is licensed for use in parathyroid surgery, does not require the administration of fluorescent contrast, which carries a risk (albeit low) of contrast allergy/anaphylaxis which was not felt to be justified in a research setting and requires no contact between the camera and tissues (as per the device used by the Mahadevan-Janssen group [9, 20]). The system, as shown in Fig. 1, was used following operative exposure of the parathyroid glands (as the brightness of AF was known to be affected by tissue covering parathyroid glands).

The primary outcomes were: (1) percentage of parathyroid glands showing an AF signal. Secondary outcomes were correlation between the intensity of parathyroid $\mathrm{AF}$ and (1) pre-operative serum calcium, (2) preoperative serum PTH, (3) SestaMIBI positivity and for glands which were excised (4) gland weight and (5) histological composition of the gland. Histology was taken as

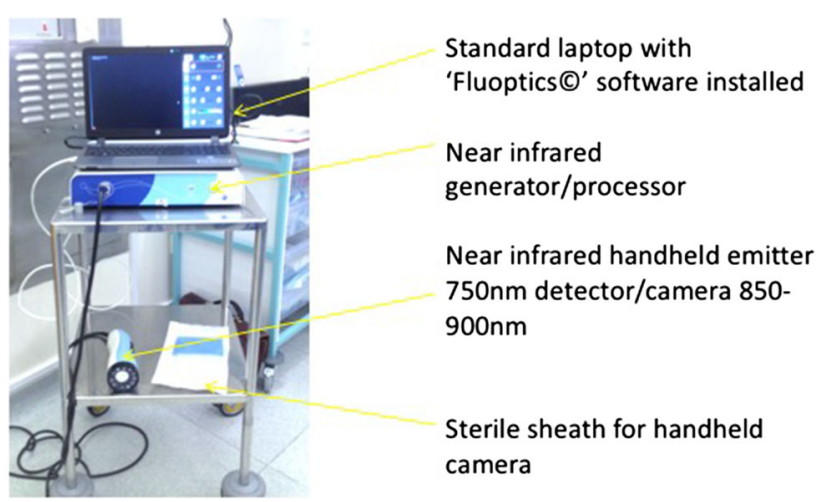

Fig. 1 'Fluobeam ${ }^{\circledR}$ 800' (Fluoptics, Grenoble, France) imaging system consisting of a standard laptop with 'Fluoptics@' software installed, a near-infrared generator/processor and a hand-held 'camera' head comprised of a combined near-infrared emitter (which produces light of $750 \mathrm{~nm}$ ) and detector (collecting light of $850-900 \mathrm{~nm}$ ). For image capture, the camera head is held in a sterile sheath by the operating surgeon at a distance of around $20 \mathrm{~cm}$ from the tissue with all ambient lights turned off 
the gold standard for identification of excised glands, whereas surgeon identification was chosen as the gold standard for parathyroids left in situ. Surgeon identification was used to avoid biopsy, which would not otherwise have been performed as part of standard surgical management, to obviate any risk to the patient solely from the study. Surgeon identification was performed by two surgeons, one of whom was a consultant surgeon (FP/NT) and one a senior trainee. The AF equipment used did not permit immediate in vivo quantification and so the intensity of parathyroid $\mathrm{AF}$ was graded as low, medium or high by the same two surgeons. This AF ranking was judged in relation to adjacent thyroid tissue with 'low' parathyroid AF being regarded as a little brighter than thyroid tissue, 'medium'much brighter and 'high' - very much brighter. Each surgeon reported their assessment to an observer (medical student) who recorded this. A flowchart of the study protocol is shown in Fig. 2.

Standard demographic information, pre- and post-operative biochemistry, results of imaging studies and histopathology including gland weight were collected. Histopathology was considered to be that of the predominant cell type, i.e. oxyphil, chief or clear, as percentage composition was not routinely reported. Statistics were calculated using SPSS (IBM Corp. Released 2016. IBM SPSS Statistics for Windows, Version 24.0. Armonk, NY: IBM Corp.). Reliability of parathyroid AF was calculated as a proportion of glands positively identified by the surgeon if not excised or histopathology if excised. The Wilcoxon signed-rank text was used to compare the continuous variables of serum calcium, PTH and weight against the ranked intensity of AF and McNemar's test to

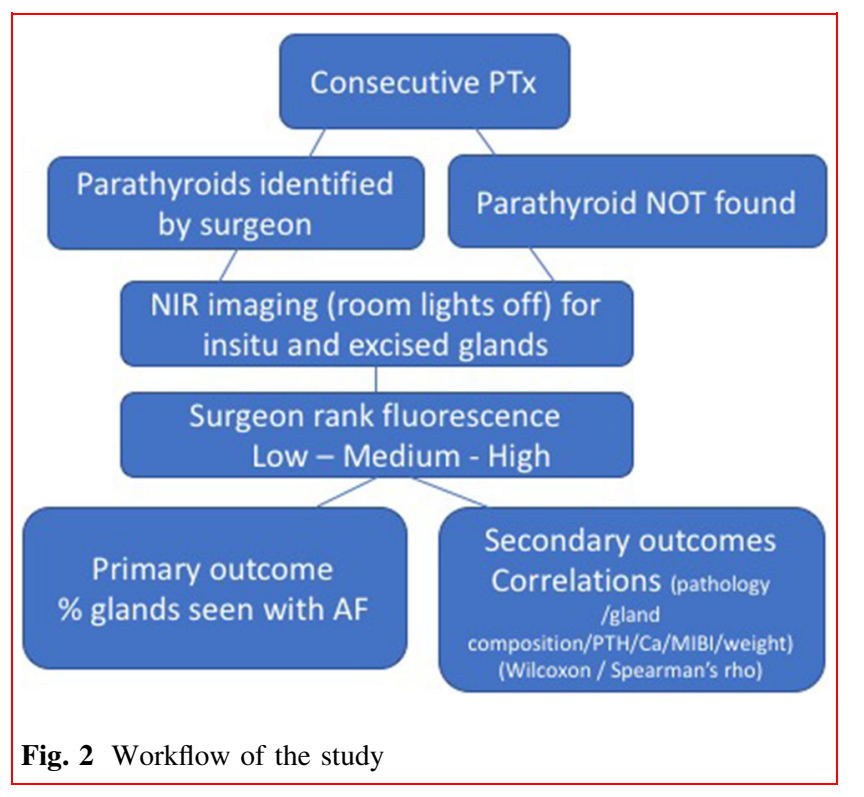

compare categorical variables of predominant composition of the excised glands against the intensity of AF.

\section{Results}

Over 8 months from February to October 2017, 96 patients underwent PTx and were included in the study. Median age was 59 years (range 24-85), almost three-quarters were female (72F:24M), 87 had pHPT and 9 secondary HPT due to renal failure. Eleven patients had undergone previous surgery for $\mathrm{pHPT}$, and 1 , previous surgery for secondary HPT (in other institutions). As shown in Table 1, in the patients with $\mathrm{pHPT}$, median pre-operative serum-corrected calcium was $2.79 \mathrm{mmol} / \mathrm{l}$ (range 2.44-3.54, upper limit of normal $=2.6 \mathrm{mmol} / \mathrm{l}$ ) and $\mathrm{PTH} 16.7 \mathrm{pmol} / \mathrm{l}$ (range 4.5-139, upper limit of normal $=6.4 \mathrm{pmol} / \mathrm{l}$ ) using Abbott Architect and IMMULITE 2000, Siemens, Llanberis, UK). Sixty-five per cent had positive SestaMIBI scans. In the renal HPT group, median pre-operative serum-corrected calcium was $2.41 \mathrm{mmol} / \mathrm{l}(2.02-2.56)$, and PTH was $241 \mathrm{pmol} / \mathrm{l}(78-571)$.

As shown in Fig. 3, there were 49 bilateral explorations, 41 unilateral and 6 focussed lateral, such that assuming the presence of 4 glands in each patient, a total of 284 glands should have been visualised at surgery. One hundred and twelve were identified and left in situ having been judged to be non-pathological and 3 glands could not be identified. A total of 172 parathyroid glands were deemed to be abnormal and excised (138 in pHPT and 34 in renal HPT). All of these were histologically confirmed to be parathyroids. Median weight of the excised parathyroids in pHPT was $0.53 \mathrm{~g}(0.05-45 \mathrm{~g})$, and on histological examination, 7 were hypercellular or large normal parathyroids and 130 abnormal, with $83 \%$ predominantly chief cell in composition. In renal HPT, all excised glands were abnormal histologically, with $88 \%$ showing chief cell predominance and $12 \%$ oxyphil.

In total, 257 parathyroids emitted $\mathrm{AF}(90.5 \%)$ and 27 (9.5\%) did not, including the 3 missing glands, which could not be identified using AF. A total of 173 glands (60.9\%) emitted a high AF signal, $61(21.5 \%)$ medium and 23 $(8.1 \%)$ low. There was a statistically significant difference in AF between the primary and secondary glands $(p<0.05)$, but no significant difference between histologically normal and abnormal glands. A statistically significant negative correlation was found between serum calcium and intensity of $\operatorname{AF}(p<0.01)$ and between serum PTH and AF in renal HPT only $(p=0.02)$. No significant correlation was found between SestaMIBI positivity ( $p=0.32$ ), gland weight $(p=0.24)$ or gland composition. In secondary HPT glands, there was a correlation between oxyphil predominance and AF $(p=0.002)$. 
Table 1 Correlations between degree of $\mathrm{AF}$ and patient/gland variables

\begin{tabular}{llll}
\hline Variable & Primary HPT & Tertiary HPT & Correlation? \\
\hline $\begin{array}{l}\text { Serum corr calcium (median } \\
\text { mmol/l) }\end{array}$ & $2.79(2.44-3.54)$ & $2.41(2.02-2.56)$ & Inverse correlation $p<0.01$ \\
Serum PTH (median pmol/l) & 16.7 & $241(78-571)$ & Inverse correlation renal glands only $p=0.02$ \\
Gland weight (median g) & 0.53 & $0.82(0.11-4.4)$ & NS $p=0.24$ \\
Predominant cell type & $83 \%$ chief cell & $88 \%$ predominantly chief and & NS EXCEPT in renal glands oxyphil-positive \\
& predominant & $12 \%$ oxyphil & correlation $p=0.002$ \\
SestaMIBI & $65 \%$ positive & Not done & NS $p=0.32$ \\
\hline
\end{tabular}

Continuous numerical variables (serum calcium, PTH, gland weight) were assessed with Wilcoxon's signed-rank and categorical data (predominant cell type on histopathology and SestaMIBI positivity) with McNemar's test

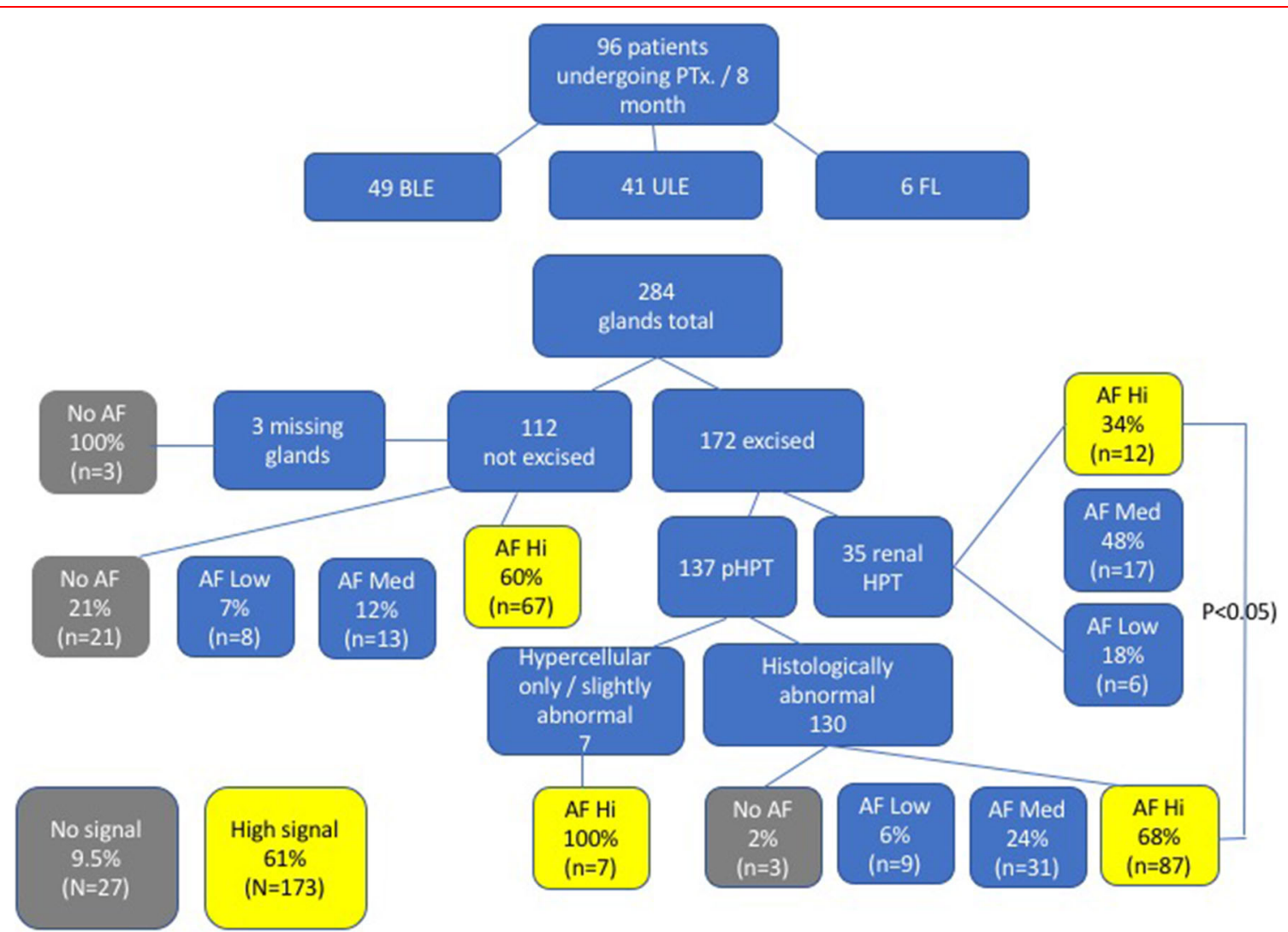

Fig. 3 Results, showing total number of glands imaged, disease process and degree of AF present. In total, 9.5\% ( $n=27)$ showed no AF signal and $61 \%(n=173)$ a high signal

\section{Discussion}

The presence of AF in just over $90 \%$ of glands in this study is equivalent to that shown by Ladurner et al. [11] in their study of 41 parathyroids sought during thyroidectomy, in which 37 showed AF (sensitivity of 90\%). However, it is lower than the $97 \%$ in 264 glands from an unselected group, in which $100 \%$ of the parathyroids in patients undergoing thyroidectomy or PTx for pHPT and 54\% in secondary HPT due to renal failure demonstrated AF [21]. This suggests that the intensity of AF may be higher in normal parathyroid tissue. Variables in this study which showed statistically significant negative correlation with $\mathrm{AF}$, i.e. serum calcium and secondary versus primary HPT, may be surrogated for the degree of abnormality of a parathyroid gland. This correlation between calcium and $\mathrm{AF}$ and the poor AF of renal-induced HPT has previously been reported by the only other study to examine the relationship between $\mathrm{AF}$ and patient/gland variables [21]. Serum PTH would also seem to reflect the degree of HPT and therefore abnormality of a gland; however, correlation with $\mathrm{AF}$ did not reach statistical significance except in the 
renal group. This was a small (9 patients, 34 glands) subgroup but may reflect the same phenomenon that more abnormal glands demonstrate less AF. However, set against that is the fact that gland weight did not correlate inversely with AF.

The absence of a correlation between positivity of SestaMIBI imaging and AF was unexpected: mitochondrial contents (NADPH) have been proposed as a fluorophore and so the authors had anticipated that brighter AF would correlate with oxyphil predominance (due to their densely packed mitochondria) and therefore SestaMIBI positivity. This relationship has not, to our knowledge, previously been tested. Similarly, a prediction that gland composition would correlate with $\mathrm{AF}$ was not manifested except in the renal HPT patients in whom oxyphil cells correlated with more intense AF. The lack of correlation with either SestaMIBI or gland composition overall may reflect which underlying fluorophore causes the AF, i.e. it may be a biological compound which is present in both chief and oxyphil cells, or in another cell type altogether. The extracellular calcium-sensing receptor has also been suggested as a possible fluorophore: it is present in highest concentrations in the chief cells and known to be downregulated in hyperparathyroidism [22], consistent with the results of other studies comparing fluorescence intensity in the parathyroids of patients with hyperparathyroidism as compared to the non-pathological glands encountered at thyroidectomy [20] and consistent with the lack of correlation with SestaMIBI in this study. The identification of the responsible fluorophore is not absolutely essential in using an AF device clinically. However, further work on the fluorophore/s and their clinical relevance may improve its performance and help to select appropriate cases for its use.

This study used a pragmatic approach to test the use of AF in a 'real-life' situation for which the Fluobeam ${ }^{\circledR}$ and other devices are designed and marketed. Although not formally assessed, the Fluobeam ${ }^{\circledR}$ was found to be simple and extremely quick to use, adding only a few minutes and the inconvenience of turning the lights down, to the operation. It also has the benefit of not requiring contact with tissues or changing the operative workflow, which may be inconvenient, time-consuming and carries a small risk of infection/tissue trauma. Care must be taken during imaging to maintain the optimum distance $(20 \mathrm{~cm})$ between device and tissues so as not to affect the AF. This distance is affected by the depth of the incision and angle at which the device is held and requires the operating surgeon to manoeuvre the device while watching the AF image onscreen until the best image is obtained [23]. With suitable positioning of the device, imaging was possible even in focused lateral surgery.
The use of surgeon identification of parathyroids as the gold standard in non-excised glands, may be subjective; however, this methodology replicates that in other studies $[10,11]$ and avoids possible harm to the patient from unnecessary biopsy of non-pathological parathyroids. The surgeon raters in this study were experienced consultants and senior trainees in a high-volume centre performing over 300 parathyroidectomies per annum. In the study, all parathyroids resected were confirmed as such on histopathology, with a cure rate of $100 \%$, and in this sample of 284 putative glands, only 3 could not be found. In fact, $\mathrm{AF}$ was not able to detect those 3 which could not be located by a surgeon. On this basis, it would seem reasonable to assume that these surgeons were a reliable standard against which to judge the clinical utility of AF. Surgeon ranking of AF intensity was used as the Fluobeam ${ }^{\circledR}$, like other commercially available devices $[14,15]$, and does not offer in vivo quantification. This may result in subjectivity; however, the intention of the study was to test the device performance in the setting for which it is designed, so any subjectivity in the study is a true reflection of its use in clinical practice. Ex vivo approaches have been described to quantify AF [23], but these require post hoc analysis, preventing their output from being utilised to assist with decision-making in surgery.

In this study, there were three 'missing' glands which could not be identified by the operating surgeon and were not identifiable using NIR. The use of NIR to assist with finding such glands is a very attractive target for future work. However, it is currently limited by the ability of current NIR devices to image structures hidden beneath the surface of a tissue. Improvement in the penetration of NIR imaging, even by a few millimetres, could have a significant impact on its clinical efficacy. Other areas which could be addressed include further assessment of the relationship between AF and patient/parathyroid tissue variables on larger numbers of normal and abnormal parathyroid glands to establish whether the intensity of AF is higher in more normal glands. The clinical utility of this would be to assist with surgical treatment of mild hyperparathyroidism secondary to multi-gland hyperplasia, this often being the more subtle disease and more difficult to localise intraoperatively.

In conclusion, based on the results of this study that nearly $10 \%$ of glands fail to emit an AF signal sufficient for the surgeon to recognise it and the failure to detect 3 missing glands; it is unlikely that $\mathrm{AF}$ will have any meaningful impact on cure rates in HPT. Its routine use in clinical practice for HPT therefore cannot be justified. However, further improvements in the technology, such as depth of imaging, and confirmation of the relationship between AF intensity and normal parathyroid tissue, may 
in future allow NIR imaging to be of assistance in select cases.

Funding This study was funded by the Butterfly Thyroid Cancer Trust and a contribution from Mr. James Salter (Grant No. 0317). The work was presented in the prize session of the European Society of Endocrine Surgeons' meeting in Amsterdam, May 2018.

\section{Compliance with ethical standards}

Conflict of interest The authors have no conflicts of interest to declare.

Informed consent Informed consent was gained from all individual participants in the study.

Open Access This article is distributed under the terms of the Creative Commons Attribution 4.0 International License (http://crea tivecommons.org/licenses/by/4.0/), which permits unrestricted use, distribution, and reproduction in any medium, provided you give appropriate credit to the original author(s) and the source, provide a link to the Creative Commons license, and indicate if changes were made.

\section{References}

1. Croce AC, Bottiroli G (2014) Autofluorescence spectroscopy and imaging: a tool for biomedical research and diagnosis. Eur $\mathbf{J}$ Histochem 58(4):2461

2. Meerwaldt R, Lutgers HL, Links TP, Graaff R, Baynes JW, Gans ROB et al (2007) Skin autofluorescence is a strong predictor of cardiac mortality in diabetes. Diabetes Care 30(1):107-112

3. Tanaka K, Tani Y, Asai J, Nemoto F, Kusano Y, Suzuki H et al (2005) Skin autofluorescence is associated with severity of vascular complications in Japanese patients with Type 2 diabetes. Diabet Med 16(12):3687-3693

4. Keller MD, Majumder SK, Kelley MC, Meszoely IM, Boulos FI, Olivares GM et al (2010) Autofluorescence and diffuse reflectance spectroscopy and spectral imaging for breast surgical margin analysis. Lasers Surg Med 42(1):15-23

5. Won Y, Hong SH, Yu HY, Kwon YH, Yun SJ, Lee SC et al (2007) Photodetection of basal cell carcinoma using methyl 5-aminolaevulinate-induced protoporphyrin IX based on fluorescence image analysis. Clin Exp Dermatol 32(4):423-429

6. Kuwayama K, Miyauchi S, Tateoka R, Abe H, Kamo N (2002) Fluorescein uptake by a monocarboxylic acid transporter in human intestinal Caco-2 cells. Biochem Pharmacol 63(1):81-88

7. Ris F, Hompes R, Cunningham C, Lindsey I, Guy R, Jones O et al (2014) Near-infrared (NIR) perfusion angiography in minimally invasive colorectal surgery. Surg Endosc Other Interv Tech 28(7):2221-2226

8. Vahrmeijer AL, Hutteman M, Van Der Vorst JR, Van De Velde CJH, Frangioni JV (2013) Image-guided cancer surgery using near-infrared fluorescence. Nat Rev Clin Oncol 10(9):507-518

9. Paras C, Keller M, White L, Phay J, Mahadevan-Jansen A (2011) Near-infrared autofluorescence for the detection of parathyroid glands. J Biomed Opt 16(6):67012
10. McWade MA, Paras C, White LM, Phay JE, Mahadevan-Jansen A, Broome JT (2013) A novel optical approach to intraoperative detection of parathyroid glands. Surgery (United States) 154(6):1371-1377

11. Ladurner R, Al Arabi N, Guendogar U, Hallfeldt K, Stepp H, Gallwas J (2018) Near-infrared autofluorescence imaging to detect parathyroid glands in thyroid surgery. Ann R Coll Surg Engl 100(1):33-36

12. Mittendorf EA, McHenry CR (2004) Complications and sequelae of thyroidectomy and an analysis of surgeon experience and outcome. Surg Technol Int 12:152-157

13. Stavrakis AI, Ituarte PHG, Ko CY, Yeh MW (2007) Surgeon volume as a predictor of outcomes in inpatient and outpatient endocrine surgery. Surgery 142(6):887-899

14. Vidal Fortuny J, Belfontali V, Sadowski SM, Karenovics W, Guigard S, Triponez F (2016) Parathyroid gland angiography with indocyanine green fluorescence to predict parathyroid function after thyroid surgery. Br J Surg 103(5):537-543

15. Vidal Fortuny J, Karenovics W, Triponez F, Sadowski SM (2016) Intra-operative indocyanine green angiography of the parathyroid gland. World J Surg. https://doi.org/10.1007/s00268-016-3493-2

16. Christensen SE, Nissen PH, Vestergaard P, Heickendorff L, Brixen K, Mosekilde L (2008) Discriminative power of three indices of renal calcium excretion for the distinction between familial hypocalciuric hypercalcaemia and primary hyperparathyroidism: a follow-up study on methods. Clin Endocrinol (Oxf) 69(5):713-720

17. Bilezikian JP, Brandi ML, Eastell R, Silverberg SJ, Udelsman R, Marcocci C et al (2014) Guidelines for the management of asymptomatic primary hyperparathyroidism: Summary statement from the fourth international workshop. J Clin Endocrinol Metab 99(10):3561-3569

18. Wheeler DC, Winkelmayer WC (2017) KDIGO 2017 clinical practice guideline for the diagnosis, evaluation, prevention and treatment of Chronic Kidney Disease-Mineral and Bone Disorder (CKD-MBD). Kidney Int Suppl 7(1):1-59

19. Carneiro DM, Solorzano CC, Nader MC, Ramirez M, Irvin GL, Udelsman R et al (2003) Comparison of intraoperative iPTH assay (QPTH) criteria in guiding PTx: which criterion is the most accurate? Surgery 134:973-981

20. McWade MA, Paras C, White LM, Phay JE, Solórzano CC, Broome JT et al (2014) Label-free intraoperative parathyroid localization with near-infrared autofluorescence imaging. J Clin Endocrinol Metab 99(12):4574-4580

21. McWade MA, Sanders ME, Broome JT, Solórzano CC, Mahadevan-Jansen A (2016) Establishing the clinical utility of autofluorescence spectroscopy for parathyroid detection. Surgery (United States) 159:193-202

22. Gogusev J, Duchambon P, Hory B, Giovannini M, Goureau Y, Sarfati E et al (1997) Depressed expression of calcium receptor in parathyroid gland tissue of patients with hyperparathyroidism. Kidney Int 51(1):328-336

23. Shinden Y, Nakajo A, Arima H, Tanoue K, Hirata M, Kijima Y et al (2017) Intraoperative identification of the parathyroid gland with a fluorescence detection system. World J Surg 41(6):1506-1512. https://doi.org/10.1007/s00268-017-3903-0

Publisher's Note Springer Nature remains neutral with regard to jurisdictional claims in published maps and institutional affiliations. 